\title{
LED Excitation of an Imaging Cytometer for Bead-based Immunoassay
}

\author{
Xilong Yuan, Todd Darcie, Jonathan J.D. McKendry, Martin D. Dawson, Fellow, IEEE, Michael J. \\ Strain, Member, IEEE, J Stewart Aitchison, Fellow, IEEE
}

Abstract-We present and demonstrate a light-emitting diod $\$ 3$ (LED)-excited imaging cytometer for the detection of bead-based 4 immunoassay samples. A broad area green LED illuminates th 45 specimen plane using a set of aspheric lenses and an excitation 6 filter. The imaging module was comprised of an objective lens, filters, tube lens, and camera. To demonstrate the multiplex 77 capability of the presented system, a panel of three sets of bead\$ 8 with varying classification fluorescence intensity was employed 9 Experimental results revealed that the LED light source provides 0 uniform illumination across the specimen plane, and therefore 1 permits the multiplex detection of three biomarkers. Detection of a sepsis biomarker, procalcitonin, was used to demonstrate the detection sensitivity and measurement range of the system. The 3 imaging cytometer can detect the concentration of procalcitonin a low as $24.4 \mathrm{pg} / \mathrm{mL}$ and it holds the potential for being develope $\overline{5}$ for point-of-care testing applications.

Index Terms - biomarkers, biosensors, cytometer, fluorescence 8 microscopy, light emitting diodes.

\section{INTRODUCTION}

(e ELL and protein biomarkers are commonly tested in2 disease diagnosis and monitoring [1-4]. Flow cytometry is 83 one of the workhorses of cell and protein biomarker testing 64 however, conventional flow cytometers have large form factor 85 and are also expensive to acquire and maintain. Therefore, onl $\$ 6$ centralized laboratories and hospitals are equipped with floy 7 cytometry analyzers. This results in significant measuremenf8 turn-around times and a lack of access to laboratory testing ig9 resource-limited settings.

These issues can be addressed through use of a 'portable 1 cytometer' technology. Our group reported a portable 2 CD4/CD8 cell counting device for HIV patient monitoring $\left[5_{73}\right.$ 7]. Recently, we also reported the development of Dengue 4 antibody / antigen detection system using bead-based sandwich 5 immunoassay [8]. Other researchers reported the improvemen $\$ 6$ of a variety of components of the cytometer systems, includin $\$ 7$ microfluidic sample handling, lens-free microscopy, an $\$ 8$ detection/signal processing [2,4,9-12]. Miniaturized flow9 cytometers have been developed for a variety of applications 80 including pathogen identification, cellula\&1

Xilong Yuan, Todd Darcie, and J Stewart Aitchison are with the Department of Electrical and Computer Engineering, University of Toronto, Toronto, Canada M5S 3G4 (e-mail: xilong.yuan@utoronto.ca; todd.darcie@mail.utoronto.ca; stewart.aitchison@utoronto.ca)

Jonathan J.D. McKendry, Martin D. Dawson, and Michael J. Strain are with the Department of Physics, Institute of Photonics, University of Strathclyde, immunophenotyping, multiplex biomarker analysis, and rare cell analysis and sorting [13-16]. Because of the value of portable cytometers for point-of-care applications, this field continues to attract attention from academic research and industrial communities.

Conventional flow cytometers use a sheath fluid to hydrodynamically focus the target cells/particles in a single-file stream. Laser sources are aligned to strike the target cells/particles in the focused stream. The "imaging cytometry" approach we adopted is based on the principle of fluorescence microscopy. Samples containing cells/particles are introduced into a shallow and wide microfluidic channel, followed by fluorescence imaging and image processing. The imaging cytometry approach could potentially be developed into a smaller form factor because hydrodynamic focusing is not required in the design. Nonetheless, the imaging cytometry approach requires a uniform illumination profile across the microscopic field of view. The uniformity of the illumination intensity is critical for achieving the accuracy and precision of quantitative fluorescence detection.

In our previous research, lasers were used for the illumination of the imaging system [8] where the Gaussian intensity profile results in a non-uniform illumination of the micro-fluidic channel. With the development of GaN LEDs over the last 20-30 years, LEDs are now available with high brightness and efficiency, with wavelengths that cover from the deep ultraviolet to the near infrared, providing flexibility in matching suitable LEDs with the requirements of fluorophores. LEDs are compact, robust, and can be powered by low-voltage batteries or relatively inexpensive switchable power supplies making them particularly well-suited to point-of-care applications [17].

Bead-based immunoassays are an important application of flow cytometers for protein biomarker detection [18,19]. Polystyrene beads are typically utilized as the substrate for binding with target molecules and therefore converting the chemical information (biomarker concentration) into an optical signal (fluorescence intensity). As shown in Step a in Fig. 1, polystyrene beads are encoded with different concentration levels of a red fluorophore (e.g. allophycocyanin). Beads with

Glasgow G1 1RD, U.K. (e-mail: jonathan.mckendry@strath.ac.uk; m.dawson@strath.ac.uk; michael.strain@strath.ac.uk).

JJDM, MS and MDD acknowledge EPSRC funding under grant EP/M01326X/1." 
1 unique fluorescence intensity signature are thus generated, sucB8 2 as A, B and C in Fig. 1. Therefore, the red fluorescence intensity9 3 could provide bead classification information. The second ste 0 4 is to prepare a capture antibody on the individual bead sets 1 5 Afterwards, a panel of bead sets reacts with the specimen (e.g42 6 blood), captures biomarkers selectively, and generates 43 7 reporter fluorescence from a yellow fluorophore (e.g44 8 phycoerythrin). Because the intensity of the reportef5 9 fluorescence is correlated with the concentration of a biomarke 6 10 molecule, we could measure the concentration of a biomarkef 7 11 by analyzing the yellow fluorescence intensity. Combing th $₫ 8$

multiplex capacity of the classification fluorescence, bead49 based immunoassay could typically detect $5-10$ biomarkers ino one run. By exciting the beads with an excitation LED 1 fluorescent images can be generated for target protein 2 concentration analysis.

In this paper, we propose and experimentally demonstrate th 64 detection of a sepsis biomarker called procalcitonin using th 55 bead-based immunoassay approach. An LED-excited imaging6 cytometer prototype is presented based on the principle of a67 infinity-corrected fluorescence microscope. A green LEJ8 (center wavelength $520 \mathrm{~nm}$ ) is used as the light source. A pane 9 of three sets of beads was used to demonstrate the feasibility o 60 bead multiplexing. The concentration of procalcitonin is fitte 61 against the fluorescence intensity to demonstrate the detection 2 performance of the prototype imaging cytometer system.

A schematic of the LED-excited imaging cytometer 66 prototype is shown in Fig. 2A. It consists of three modules: th 67 LED light source, microfluidic sample cartridge, and imaging 68 module.

A green LED with $520 \mathrm{~nm}$ peak wavelength (Lumiled $^{0}$ LXZ1-PM01) was used to excite the two fluorophores 1 (allophycocyanin, 'APC', and phycoerythrin, 'PE'). The 22 individually packaged LED die is mounted on a PCB and ${ }^{3}$ controlled using a Field-Programmable Gate Array (FPGA 74 chip and MATLAB code. Two identical aspheric lenses 5

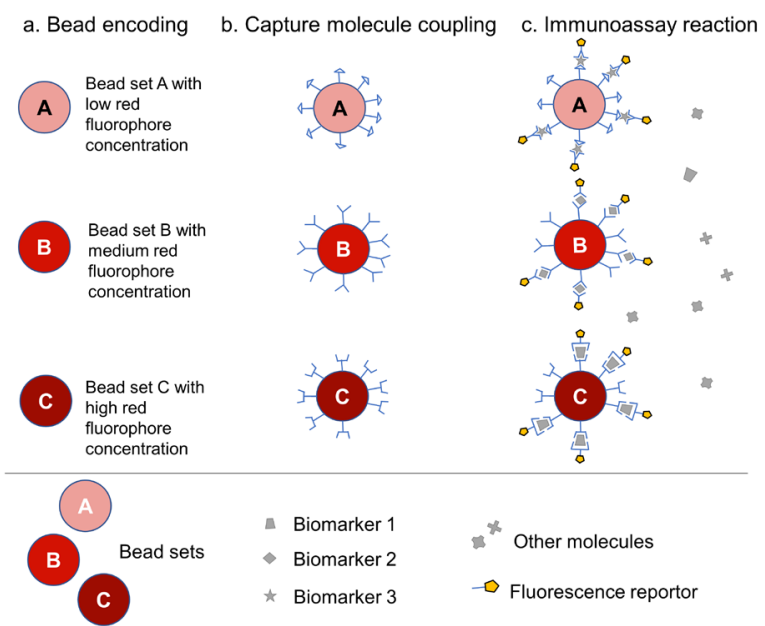

Fig. 1. Schematic representation of the steps in a bead-based immunoassay91 for the simultaneous detection of three biomarkers, a) bead encoding, b) resulting functionalized bead and c) the final bead with captured biomarker 92 and fluorescence reporter molecule.
(Thorlabs ACL2520U, Ø25mm, F=20mm, NA=0.60) were used to project the LED light to the specimen plane.

One of the challenges of using LED illumination is the crosscoupling of the LED emission into the fluorescent channels. As shown in Fig. 1S in the supporting document, the green LED has a peak wavelength of $520 \mathrm{~nm}$, but the broadband LED emission extends beyond $550 \mathrm{~nm}$ and overlaps with the emission band of the PE reporter emission, as shown in Fig. 2S in the supporting document. An excitation filter (Semrock FF01-457/530/628) with an optical density of $>6$ is used to block the green LED emission and hence isolate the fluorescent bands. Since the filters only achieve the required optical density under normal incidence, the excitation filter is placed in the columnated beam between the two aspheric lenses.

A 3D printed cartridge was used to hold the microfluidic sample chip, allowing for the alignment with light source and the imaging system. The green LED die can illuminate the entire area $(1 \mathrm{~mm} \times 1 \mathrm{~mm})$ of the specimen plane with an estimated optical power of $16 \mathrm{~mW} / \mathrm{mm}^{2}$. A microfluidic chip with wide $(800 \mu \mathrm{m})$ and shallow $(20 \mu \mathrm{m})$ channels was used to confine the sample. This configuration produces a ribbon-like fluid sample that increases throughput while limiting the chance of multiple beads flowing through the same location at the same time.

The imaging module was comprised of an infinity-corrected optical system. The fluorescence emitted at the specimen plane was collimated by a $10 \mathrm{x}$ objective lens and focused onto the sensor plane using a $200 \mathrm{~mm}$ tube lens. A dual bandpass filter (center wavelengths $577 \mathrm{~nm}$ and $690 \mathrm{~nm}$ ) was used to isolate the emission fluorescence from the two fluorophores as well as block excitation light from the green LED. Additionally, a bandpass filter with a $565 \mathrm{~nm}$ center wavelength and a longpass filter with $665 \mathrm{~nm}$ cut-on wavelength are assembled on flip mounts, allowing us to take separate images of the classification and reporter fluorescence channel. A pco.edge 4.2 LT monochrome scientific camera is used in the prototype. Using this combination of filters resulted in a pump background of 143.8 (16 bit) compared to a noise floor of 100.5 for the CMOS sensor.

To characterize the intensity profile of the LED light on the specimen plane, multiple neutral density filters (total OD 5.0) are applied to take an image of the green LED profile on the specimen plane. The LED relative intensity across the specimen plane has coefficients of variation of $4.2 \%$ and $2.2 \%$ for the center $\mathrm{x}$ profile and the center $\mathrm{y}$ profile, respectively. In comparison, a gaussian laser beam was determined to have coefficients of variation of $52.3 \%$ and $55.9 \%$ for the center $\mathrm{x}$ profile and the center y profile, respectively. Therefore, it is considered satisfactory to use the LED excitation for further fluorescent imaging testing.

\section{IMAGE PROCESSING AND BEAD CLASSIFICATION}

In the current imaging cytometer prototype, a $565 \mathrm{~nm}$ bandpass filter and a $650 \mathrm{~nm}$ long-pass filter are switched when capturing fluorescent images from the two fluorophores, classification fluorophore APC, and reporter fluorophore PE. Representative images of the classification fluorescence 


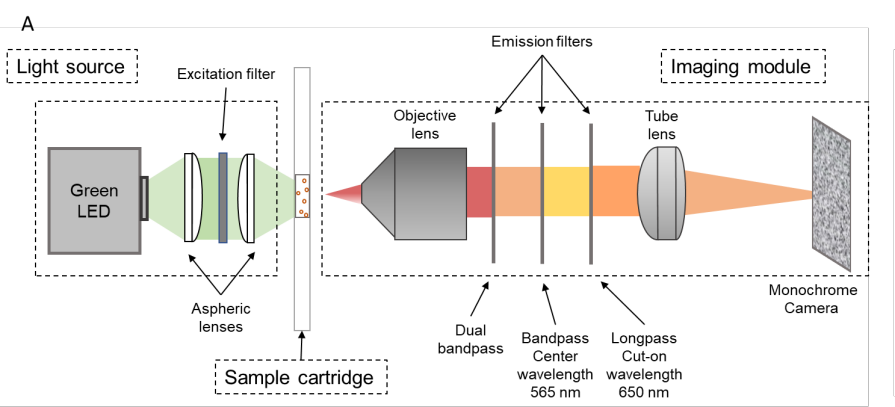

B

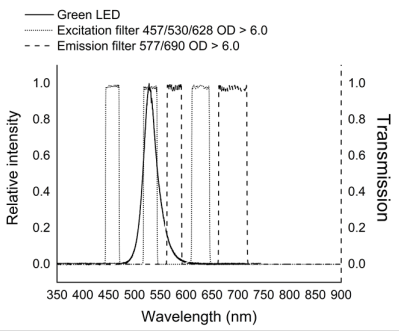

C

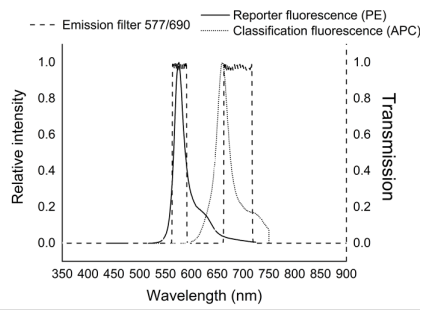

Fig. 2. (A) Schematic of the LED-excited imaging cytometer prototype. (B) Spectra of the green LED, excitation filter and emission filter. (C) Spectra of the classification fluorophore (APC), the reporter fluorophore (PE), and the dual bandpass emission filter.

1 channel and reporter fluorescence channel are shown in Fig. 344 2 and $3 \mathrm{~B}$, respectively. Images in Fig. $3 \mathrm{~A}$ and $3 \mathrm{~B}$ represents th $\$ 5$ 3 same group of beads, captured using the individual fluorescent6 4 filters. Therefore, the bead image in Fig. 3A comes from the re 47 5 classification fluorophore APC. The bead image in Fig. 3B8 6 comes from the yellow fluorophore PE for biomarke 9 7 concentration detection. The captured images are processed in 0 8 Image J by defining the region of interest (ROI) and analyzing 1 9 fluorescence intensity of the ROI, as shown in Fig. 3A and 3B52

To compare the uniformity of LED illumination with a lase 3 beam, a representative set of beads with a consistent rea4 fluorophore concentration was used with both LEI5 illumination, or a green laser (Laserglow, LRS-0532). It was6 found that the LED illumination showed significant decrease of7 fluorescence intensity variation $(\mathrm{CV}=22.2 \%)$ compared with $\mathbf{5 8}$ laser illumination source $(\mathrm{CV}=53.7 \%)$. The decrease of 9 fluorescence intensity variation indicates the improve 60 illumination uniformity using the demonstrated LED light 1 source. The improved illumination uniformity permits th62 multiplex detection of biomarkers. Because classification3 fluorescence peak overlapping can be reduced, classificatio 64 accuracy and multiplexing capability can be improved.

To demonstrate the multiplexing capability of the bead-base 66 immunoassay (shown in Fig. 1), the prototype cytometer wa67 used to capture images of a panel of three sets of beads witk 8 different concentration of red fluorophore (using the $650 \mathrm{~nm} 6$ long-pass filter). Bead fluorescence intensity in th $\approx 0$ classification channel is plotted in histogram to show the 1 distribution of classification fluorescence intensity. As show 2 in Fig. 4a, the fluorescence intensities of bead set A, B, and 73 show three clearly separate peaks, indicating that the three bea $\mathbb{4} 4$ sets can be classified. There are some ROIs that showed5 fluorescence intensities between the peaks. Therefore, narrower6 intervals of classification fluorescence are defined for the7 individual bead sets, to reduce the probability of8 misclassification (Fig. 4a).

\section{REPORTER FLUORESCENCE AND BIOMARKER QUANTITATION}

After analyzing the classification fluorescence channel, w 83 investigate the biomarker detection capability of the prototyp 4 cytometer by analyzing the reporter fluorescence image $\$ 5$ captured using the $565 \mathrm{~nm}$ bandpass filter. Similar technique $\$ 6$ were adopted to define the ROIs and generate reporter fluorescence intensities from the ROIs (Fig. 3B).

Procalcitonin is a sepsis biomarker that has clinical significance for diagnosing the severity of sepsis. A cut-off concentration lower than $0.5 \mathrm{ng} / \mathrm{mL}$ is indicative of absence of infection, or a mild infection. In patients with severe sepsis complications and less likelihood of survival, procalcitonin levels could reach $10 \mathrm{ng} / \mathrm{mL}$ or higher [20]. In this research, a bead-based immunoassay of procalcitonin is developed by coupling capture antibodies specific to procalcitonin using an approach reported earlier [8]. The coupled beads react with serial dilutions of procalcitonin standards $(6.1-25000 \mathrm{pg} / \mathrm{mL})$ spiked in buffer solutions by forming an antigen-antibody complex, followed by binding with detection antibody and reporter fluorophore PE. An empirical approach for determining limit of detection (LoD) was adopted by running procalcitonin standard samples with very low concentrations, e.g. $6.1 \mathrm{pg} / \mathrm{mL}$, and $24.4 \mathrm{pg} / \mathrm{mL}$.

After the bead-based immunoassay reaction, the bead samples are introduced into the microfluidic chip for fluorescent imaging. Multiple images of beads were captured for each immunoassay sample to collect enough number of ROIs (e.g. 30) for statistical analysis of median fluorescence intensity (refer to supporting document Fig. 3S). Integrated intensity of ROIs is used for fluorescence intensity analysis. As shown in Fig. 4b, median fluorescence intensity is fitted with procalcitonin concentration using a four-parameter logistic regression model. The coefficient of determination $\left(\mathrm{R}^{2}\right)$ is calculated as 0.999 . This indicates a good fit between the output response (fluorescence intensity) and the input parameter (concentration). The measurement range of the procedure is determined to be $24.4-25000 \mathrm{pg} / \mathrm{mL}$, indicating a 3-decade dynamic range. The LED-excited imaging cytometer can detect the procalcitonin concentration as low as $24.4 \mathrm{pg} / \mathrm{mL}$ (LoD). Although this is not as sensitive as $6.1 \mathrm{pg} / \mathrm{mL}$ determined using a conventional flow cytometer, both sensitivity levels are below the cut-off concentration of procalcitonin for sepsis diagnosis, indicating the potential for meeting the sensitivity requirements of clinical relevance. Comparing this result with a previous study using a Gaussian beam laser, we found an improvement of sensitivity from $\mathrm{ng} / \mathrm{mL}$ level to $\mathrm{pg} / \mathrm{mL}$ level [8,21]. This is partially due to the decreased variation of bead fluorescence intensity resulting from the uniform LED illumination, although a more sensitive assay protocol may also contribute to 
A

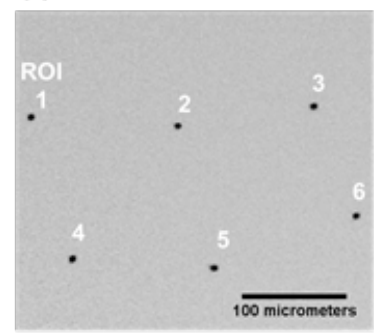

Classification fluorescence image

\section{B}

Reporter fluorescence image

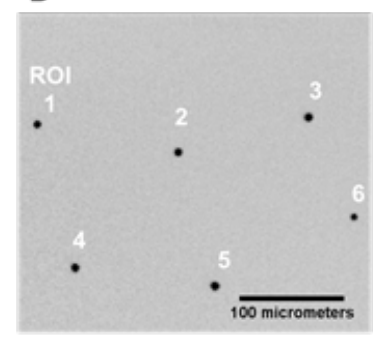

27

28

29

30

31

32

33

34

35

36

37
38

Fig. 3. Representative images (inverted) of a population of beads from (A) the 39 classification fluorescence channel and (B) the reporter fluorescence channel.40 A $565 \mathrm{~nm}$ bandpass filter and a $650 \mathrm{~nm}$ long-pass filter are switched when 41 capturing the individual fluorescent images.

1 the improved detection sensitivity.

In this study, we proved that three sets of beads can b 45 classified in a multiplexed format. PCT is used to demonstrat the analytical performance of the LED-excited imagin $\$ 8$ 5 cytometer prototype. Therefore, further studies will b $\$ 9$ conducted to investigate the bead-based immunoassay of 0 multiple protein biomarkers, and to test the detectio ${ }_{2}$ performance (sensitivity, accuracy, and precision) of th $\$ 3$ multiplex immunoassay. Oblique and epifluorescence desig 54 may be implemented to further improve the optical sensitivity 55 Additional studies are also needed to integrate the bead-base 77 immunoassay in a microfluidic sample cartridge for automate 8 sample preparation. Image capturing and processing is 50 performed manually in this work. Therefore automation iø 1 required to demonstrate the functionality of the unit in the field 22 The prototype may be used as a model to develop into a field 63 deployable system that can be validated in clinics or remot 85 communities for blood testing and clinical diagnosis.

The presented LED-excited imaging cytometer system was 71 characterized for illumination uniformity and used for th 92 detection of a panel of three sets of beads. Detection of a sepsiz3 biomarker is presented and showed good detection sensitivity 75 and accuracy. This demonstrated that LED illumination is 95 promising approach for imaging cytometer and its applications77 The presented cytometer prototype can be further developea 8 a

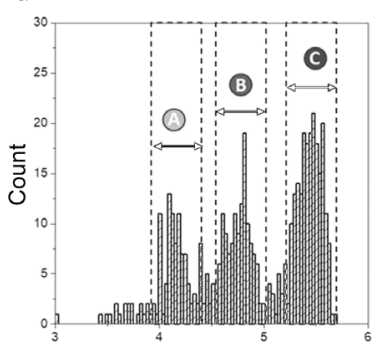

ROI Intensity (Log10) b

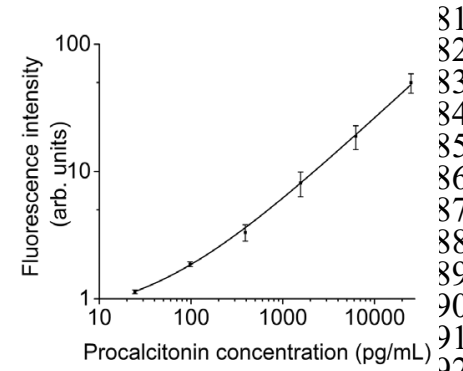

Fig. 4. (a) Histogram of a panel of three sets of beads (region of interests, 93 ROI) with different red fluorophore concentrations. Double arrows showed the 94 classification intervals of the bead set A, B, and C. (b) Calibration curve of 95 procalcitonin concentration using median reporter fluorescence intensity. 96 Four-parameter logistic model is used for curve fitting, and $\mathrm{R}^{2}$ is 0.999 . Error 97 bars represents the variance of median fluorescence intensity for multiple 98 frames of captured images $(n \geqslant 3)$. into a point-of-care testing device for disease diagnosis and monitoring.

\section{REFERENCES}

[1] Z. Yu, G. Cai, X. Liu, and D. Tang, "Pressure-Based Biosensor Integrated with a Flexible Pressure Sensor and an Electrochromic Device for Visual Detection," Anal Chem, vol. 93, no. 5, pp. 2916-2925, Feb 92021.

[2] S. Zhang, Z. Li, and Q. Wei, "Smartphone-based cytometric biosensors for point-of-care cellular diagnostics," Nanotechnology and Precision Engineering, vol. 3, no. 1, pp. 32-42, 2020.

[3] Z. Yu, Y. Tang, G. Cai, R. Ren, and D. Tang, "Paper Electrode-Based Flexible Pressure Sensor for Point-of-Care Immunoassay with Digital Multimeter," Anal Chem, vol. 91, no. 2, pp. 1222-1226, Jan 152019.

[4] K. Ming et al., "Integrated quantum dot barcode smartphone optical device for wireless multiplexed diagnosis of infected patients," ACS Nano, vol. 9, no. 3, pp. 3060-74, Mar 242015.

[5] J. Dou, L. Chen, R. Nayyar, and J. S. Aitchison, "A miniaturized particle detection system for HIV monitoring," in 2013 IEEE Photonics Conference, 2013: IEEE, pp. 5-7.

[6] J. Dou, L. Chen, R. Nayyar, and S. Aitchison, "A microfluidic based optical particle detection method," in Optical Diagnostics and Sensing XII: Toward Point-of-Care Diagnostics; and Design and Performance Validation of Phantoms Used in Conjunction with Optical Measurement of Tissue IV, 2012, vol. 8229: International Society for Optics and Photonics, p. 82290B.

[7] M. M. Gong and D. Sinton, "Turning the page: advancing paper-based microfluidics for broad diagnostic application," Chemical reviews, vol. 117, no. 12, pp. 8447-8480, 2017.

[8] X. Yuan et al., "Bead-based multiplex detection of dengue biomarkers in a portable imaging device," Biomed Opt Express, vol. 11, no. 11, pp. 61546167,2020

[9] D. Barat, D. Spencer, G. Benazzi, M. C. Mowlem, and H. Morgan, "Simultaneous high speed optical and impedance analysis of single particles with a microfluidic cytometer," Lab Chip, vol. 12, no. 1, pp. 118-126, 2012. [10] M. E. Piyasena and S. W. Graves, "The intersection of flow cytometry with microfluidics and microfabrication," Lab Chip, vol. 14, no. 6, pp. 10441059, 2014.

[11] Y. Wu and A. Ozcan, "Lensless digital holographic microscopy and its applications in biomedicine and environmental monitoring," Methods, vol. 136, pp. 4-16, 2018.

[12] Z. Göröcs et al., "Label-free detection of Giardia lamblia cysts using a deep learning-enabled portable imaging flow cytometer," Lab Chip, vol. 20, no. 23, pp. 4404-4412, 2020.

[13] G. Stybayeva et al., "Lensfree holographic imaging of antibody microarrays for high-throughput detection of leukocyte numbers and function," Analytical chemistry, vol. 82, no. 9, pp. 3736-3744, 2010. [14] D. K. Singh, C. C. Ahrens, W. Li, and S. A. Vanapalli, "Label-free, high-throughput holographic screening and enumeration of tumor cells in blood," Lab Chip, vol. 17, no. 17, pp. 2920-2932, 2017.

[15] B. Nasseri, N. Soleimani, N. Rabiee, A. Kalbasi, M. Karimi, and M. R. Hamblin, "Point-of-care microfluidic devices for pathogen detection," Biosensors and Bioelectronics, vol. 117, pp. 112-128, 2018.

[16] Y. Lee, B. Kim, and S. Choi, "Integrated microflow cytometry for portable immunophenotypic cell analysis," Sensors and Actuators A: Physical, p. $112038,2020$.

[17] H. S. Wasisto, J. D. Prades, J. Gülink, and A. Waag, "Beyond solidstate lighting: Miniaturization, hybrid integration, and applications of GaN nano-and micro-LEDs," Applied Physics Reviews, vol. 6, no. 4, p. 041315, 2019.

[18] H. Graham, D. J. Chandler, and S. A. Dunbar, "The genesis and evolution of bead-based multiplexing," Methods, vol. 158, pp. 2-11, Apr 1 2019.

[19] W. Kong et al., "Magnetic microspheres-based cytometric bead array assay for highly sensitive detection of ochratoxin A," Biosens Bioelectron, vol. 94, pp. 420-428, Aug 152017.

[20] M. Stocker, M. Fontana, S. El Helou, K. Wegscheider, and T. M. Berger, "Use of procalcitonin-guided decision-making to shorten antibiotic therapy in suspected neonatal early-onset sepsis: prospective randomized intervention trial," Neonatology, vol. 97, no. 2, pp. 165-174, 2010.

[21] X. Yuan, T. Darcie, J. S. Aitchison, J. J. McKendry, M. J. Strain, and M. D. Dawson, "LED excitation of an on-chip imaging flow cytometer for bead-based immunoassay," in 2020 IEEE Photonics Conference (IPC): IEEE, pp. $1-2$. 